\title{
Extending the duration of response in chronic myelogenous leukemia: targeted therapy with sequential tyrosine kinase inhibitors
}

\author{
Michael G. Martin · John F. DiPersio • \\ Geoffrey L. Uy
}

Received: 14 November 2008/ Accepted: 18 December 2008/Published online: 19 February 2009

(C) The Author(s) 2009. This article is published with open access at Springerlink.com

\begin{abstract}
Tyrosine kinase inhibitors (TKIs) are the mainstay for treatment of chronic myelogenous leukemia (CML). Imatinib was the first TKI approved for use in CML, but resistance to this therapy has emerged as a significant issue, and second-line options are often necessary. Increased-dose imatinib may elicit responses in some patients, but clinical evidence suggests only a minority experience sustained benefit. The second-generation TKIs, dasatinib and nilotinib, have demonstrated efficacy in patients resistant or intolerant to imatinib. Changes in therapy, with the aim of inducing durable response, should occur promptly after imatinib failure is identified as all agents are more effective in chronic phase disease than in later stages. Selection of second-line agents should be driven by efficacy and safety: dasatinib may be more effective in patients with P-loop or F359C mutations; nilotinib may be more effective in those with $\mathrm{F} 317 \mathrm{~L}$ mutations.
\end{abstract}

Keywords Dasatinib - Imatinib - Nilotinib - CML . Philadelphia chromosome $\cdot$ BCR-ABL

\section{Introduction}

Chronic myelogenous leukemia (CML) is characterized by the presence of the Philadelphia $(\mathrm{Ph})$ chromosome which encodes the BCR-ABL fusion protein, the causative molecular aberration in the pathogenesis of the disease [1]. CML is usually diagnosed in the chronic phase (CP), and,

M. G. Martin · J. F. DiPersio · G. L. Uy ( $\square)$

Section of Bone Marrow Transplantation and Leukemia,

Division of Medical Oncology, Siteman Cancer Center,

Washington University School of Medicine, Saint Louis, USA

e-mail: guy@im.wustl.edu if left untreated, the disease will progress to an accelerated phase (AP) and, ultimately, to a terminal blast phase (BP) within 3-5 years [2] (Table 1). $\mathrm{Ph}$ is also present in a subpopulation of patients with acute lymphoblastic leukemia $\left(\mathrm{Ph}^{+} \mathrm{ALL}\right)$.

Tyrosine kinase inhibitors (TKIs) which target BCR$\mathrm{ABL}$ are the mainstay for treatment of CML. Imatinib was the first approved BCR-ABL-targeted therapy for use in CML, and has substantially changed the treatment and outcomes associated with this disease. Before the introduction of such TKIs, 5-year survival rates with interferon treatment or chemotherapy were 57 and $42 \%$, respectively [6]. Imatinib therapy is associated with a 5-year overall survival rate of $89 \%$ [7]. Nonetheless, resistance has also emerged as a significant clinical issue with this agent and effective second-line and beyond treatments continue to be needed and developed. Two second-generation TKIs have been approved for the treatment of patients with imatinib resistance or intolerance. Dasatinib was approved for the treatment of patients with CML that have resistance or intolerance to imatinib. Nilotinib was approved for the treatment of patients with $\mathrm{CP}$ or AP CML who have failed prior treatment. In the age of targeted TKI therapy, it is key to select the appropriate agent at the appropriate juncture for each patient, the aim being to achieve long-term, durable responses with minimal toxicity. Here, we discuss the current treatment options for patients with CML that have failed imatinib and evaluate the important considerations when designing treatment algorithms.

\section{First-line imatinib}

In the pivotal phase III International Randomized Study of Interferon and STI571 (IRIS) trial, imatinib (400 mg/day) 
Table 1 Definitions of accelerated phase and blast crisis CML [3-5]

\begin{tabular}{|c|c|c|}
\hline & World health organization criteria & International bone marrow transplant registry criteria \\
\hline $\begin{array}{l}\text { Accelerated } \\
\text { phase }\end{array}$ & $\begin{array}{l}\text { - Peripheral blood and/or marrow blasts } 10-19 \% \\
\text { - Peripheral blood basophils } \geq 20 \% \\
\text { - Persistent thrombocytopenia }\left(<100 \times 10^{9} / \mathrm{L}\right) \text { or } \\
\text { thrombocytosis }\left(>1,000 \times 10^{9} / \mathrm{L}\right) \text { unresponsive to therapy } \\
\text { - Progressive splenomegaly and increasing white blood cell } \\
\text { count unresponsive to therapy } \\
\text { - Cytogenetic evidence of clonal evolution }\end{array}$ & $\begin{array}{l}\text { - Peripheral blood or marrow blasts } \geq 10 \% \\
\text { - Peripheral blood basophils and eosinophils } \geq 20 \% \\
\text { - Anemia or thrombocytopenia unresponsive to hydroxyurea/ } \\
\text { busulfan } \\
\text { - Persistent thrombocytosis } \\
\text { - Progressive splenomegaly } \\
\text { - Clonal evolution } \\
\text { - Peripheral blood or marrow blasts and promyelocytes } \geq 20 \% \\
\text { - Leukocyte count difficult to control with hydroxyurea/ } \\
\text { busulfan } \\
\text { - Rapid leukocyte doubling time ( }<5 \text { days) } \\
\text { - Development of myelofibrosis }\end{array}$ \\
\hline Blast crisis & $\begin{array}{l}\text { - Peripheral blood or marrow blasts } \geq 20 \% \\
\text { - Extramedullary blast proliferation } \\
\text { - Large blast foci/clusters in bone marrow biopsy }\end{array}$ & $\begin{array}{l}\text { - Peripheral blood or marrow blasts } \geq 20 \% \\
\text { - Extramedullary leukemic cell infiltrates }\end{array}$ \\
\hline
\end{tabular}

showed superior activity compared with the previous frontline therapy, interferon-a plus low-dose cytarabine, in patients with newly diagnosed CP CML. The estimated rates of major cytogenetic response (MCyR; Table 2) at 18 months were 87 and $35 \%$ with imatinib and interferon, respectively. Complete cytogenetic response (CCyR; Table 2) rates were $76 \%$ in the imatinib group and $15 \%$ in the interferon group $(P<0.001)$ [9]. At 60 months, $67 \%$ (368/553) of patients treated with imatinib achieved a CCyR, and overall survival was $89 \%$ [7].

Imatinib also has activity in patients with advanced stage CML, but the therapeutic advantages here are modest: MCyR rates recorded in phase II studies in patients with AP/BC CML were 16-24\% [10, 11]. Three-year overall survival rates for patients following imatinib failure have been reported as $72 \%$ for patients with CP CML, 30\% for patients with AP CML, and 7\% for those with $\mathrm{BC}$ disease [12]. These data underline the importance of preventing disease progression.

A recently published retrospective study in patients with CP CML receiving first-line imatinib (400-800 mg/day) has provided direct evidence that durability of response predicts patient outcome [13]. In this study of 276 patients, maintenance of a CCyR or major molecular response (MMR) for $\geq 12$ months was significantly $(P \leq 0.01)$ associated with improved progression-free survival (PFS) compared with responses lasting less than 12 months. In total, $78 \%$ of patients maintained a CCyR for $\geq 6$ months, this figure falling to $71 \%$ at $\geq 12$ months, and $54 \%$ at $\geq 24$ months. Similarly, the percentages of patients maintaining a MMR for $\geq 6, \geq 12$, and $\geq 18$ months were 54,43 , and $32 \%$, respectively. In patients receiving the currently approved first-line dosage of $400 \mathrm{mg} /$ day alone, the rates of CCyR and MMR durable for 12 months or more were lower; 59 and $39 \%$, respectively.

\section{Imatinib resistance}

Despite the impressive activity associated with imatinib therapy, resistance has emerged as a serious clinical issue. Resistance to imatinib is defined as either primary, where patients are refractory to imatinib treatment, or secondary, in which a previously achieved response is lost. In the IRIS trial, approximately $25 \%$ of patients were reported to exhibit some degree of primary resistance to imatinib: an
Table 2 Criteria for hematologic, cytogenetic, and molecular responses [8]

\begin{tabular}{|c|c|c|}
\hline $\begin{array}{l}\text { Monitoring } \\
\text { technique }\end{array}$ & $\begin{array}{l}\text { Category } \\
\text { of response }\end{array}$ & Criteria \\
\hline Hematologic & Complete & $\begin{array}{l}\text { White blood cell counts } \\
<1 \times 10^{9} / \mathrm{L} \text { plus normal differential; platelet count } \\
<450 \times 10^{9} / \mathrm{L} \text {; non-palpable spleen }\end{array}$ \\
\hline Cytogenetic & $\begin{array}{l}\text { Minor } \\
\text { Major } \\
\text { Complete }\end{array}$ & $\begin{array}{l}\mathrm{Ph}^{+} \text {metaphases }=36-95 \% \\
\mathrm{Ph}^{+} \text {metaphases }=0-35 \% \\
\mathrm{Ph}^{+} \text {metaphases }=0 \%\end{array}$ \\
\hline Molecular & Major & $\mathrm{BCR}-\mathrm{ABL} / \mathrm{ABL}$ ratio $<0.10 \%$, or $>3-\log$ decrease from baseline \\
\hline
\end{tabular}


estimated $5 \%$ of patients failed to achieve a complete hematologic response (CHR) at 3 months, $22 \%$ failed to achieve any cytogenetic response at 6 months, $23 \%$ failed to achieve at least a partial cytogenetic response (PCyR; Table 2) at 12 months, and 24\% failed to achieve a CCyR at 18 months $[7,9,14]$. Secondary resistance was also evident. After 60 months of follow-up, the estimated relapse rate was $17 \%$ and progression to AP or BP occurred in $7 \%$ of patients [7]. In a second study, intent-to-treat analysis revealed that the probability of remaining in MCyR at 5 years is $63 \%$ (i.e., $37 \%$ of patients required alternative treatment within 5 years of diagnosis) [15]. Similarly, a large retrospective analysis revealed resistance or intolerance to imatinib in $45 \%$ of patients [16]. Therefore, there is a strong clinical need for further treatment options.

Several mechanisms are likely to underlie the development of imatinib resistance. One of the most established causes of imatinib resistance is the acquisition of point mutations in the kinase domain of BCR-ABL [10]. BCR-ABL mutations have been reported at a frequency in the range of $42-90 \%$ among patients with secondary imatinib resistance [17-19]. Mutations emerge more frequently among patients with advanced disease compared with those with CP disease, and the frequency increases with disease duration [20]. Furthermore, the presence of point mutations at baseline has been shown to predict loss of CCyR on imatinib therapy [15].

Over 40 different imatinib-resistant mutations have been identified to date [21]. These generally fall within four regions of the ABL kinase domain, including the ATP binding loop (P-loop), the contact site (e.g., T315 and F317), the SH2 binding site (e.g., M351), and the A-loop [22]. The different BCR-ABL mutations emerge at varying frequencies which can differ according to the stage of the disease [21]. The most frequently occurring mutations (30-40\%) are within the P-loop. P-loop mutations confer high levels of resistance to imatinib and are associated with poor prognosis [23]. The second most frequently observed mutation is T315I [23]. This single amino acid substitution renders BCR-ABL-expressing cells insensitive to imatinib as well as other clinically available tyrosine kinase inhibitors [24]. Of note, these mutations decrease the efficacy of TKIs in CML, but do not necessarily predict an increase in the aggressiveness of the clone, and patients with mutations may have an indolent course [25].

A more recently implicated cause of imatinib resistance is the constitutive activation of downstream signaling molecules (e.g., SRC family kinases or SFKs). SFKmediated phosphorylation (i.e., activation) of BCR-ABL is required for full oncogenic activity [26]. Transfection of myeloid leukemia cells with kinase-defective HCK has been shown to block BCR-ABL-related cellular transformation [27].
Further studies indicate that SFK activation is present in imatinib-resistant CML and that such activation may be targeted therapeutically. Overexpression of the SFKs, LYN and HCK, have been reported in CML cell lines exhibiting BCR-ABL-independent imatinib resistance, and SFK inhibition in these cells resulted in growth inhibition [28, 29]. A recent report has shown that FYN (another SFK) is up-regulated by BCR-ABL and that FYN expression correlated to the stage of the disease, being significantly increased in blast crisis cells compared with chronic phase cells [30]. It is unclear to what degree and with how much hetero- or homo-geneity CML is addicted to these additional pathways.

Other proposed mechanisms affecting imatinib sensitivity include altered expression of drug influx and efflux proteins (i.e., Pgp and OCT-1) [31, 32], BCR-ABL gene amplification, and overexpression of BCR-ABL [33, 34]. In addition, many patients that develop imatinib resistance will not have a cause identified.

\section{Response-based indicators of imatinib resistance}

To ensure effective patient care in CML, the response to imatinib therapy is monitored frequently according to formally defined standards (Table 3) [35]. In this manner, resistance may be detected promptly and treatment changed, if appropriate. The National Comprehensive Cancer Network (NCCN) 2008 guidelines for CML recommend time-based landmark responses to treatment that should be met if the patient is to continue receiving the same imatinib schedule: a CHR should be achieved within 3 months, at least a minor cytogenetic response (Table 2) within 6 months, at least a MCyR within 12 months, and a CCyR within 18 months of treatment. If these landmarks are not met then a treatment change should be considered [35].

Results from the IRIS study underscore the importance of achieving such landmark responses. A retrospective analysis of outcomes of patients treated with imatinib in this trial, and those treated with interferon-a plus low-dose cytarabine in the CML91 trial demonstrated a significant survival advantage among patients who achieved a MCyR by 12 months, irrespective of the treatment administered [36]. Similarly, CCyR was found to be an independent predictor of survival and the key prognostic indicator in CML [8, 36, 37]. There are a number of prerequisites for gaining a CCyR. A hematologic response is a prerequisite for attaining a CCyR and also for long-term survival [38]. The degree of preceding cytogenetic response is also crucial. In the IRIS trial, the probability of eventually achieving a CCyR was only $15 \%$ if the karyotype at 6 months was $>95 \% \mathrm{Ph}$ chromosome-positive. Furthermore, if the response after 12 months of treatment was less 
Table 3 NCCN monitoring guidelines for patients with CML receiving TKI therapy [35]

\begin{tabular}{ll}
\hline Time point of response & Monitoring measures \\
\hline Diagnosis & - Bone marrow cytogenetics (or FISH analysis of peripheral blood) \\
& $\bullet$ Measurement of BCR-ABL transcript levels \\
During apparent response to therapy & - Measurement of BCR-ABL transcript levels every 3 months \\
& - Bone marrow cytogenetic analysis at 6 and 12 months, and at 18 months if CCyR \\
& not achieved by 12 months \\
At CCyR & - Measurement of BCR-ABL transcript levels every 3 months \\
& - Bone marrow cytogenetic analysis every $12-18$ months in case of clonal abnormalities \\
Treatment failure, or during AP/BC stage CML & - BCR-ABL mutation analysis \\
Rising ( $\geq 1 \log )$ BCR-ABL transcript levels & $\bullet$ Repeat measurement of BCR-ABL transcript levels in 1 month \\
& $\bullet$ Monthly measurement of BCR-ABL transcript levels, if rise confirmed \\
& $\bullet$ Consider BCR-ABL mutation analysis
\end{tabular}

than a MCyR, the probability of achieving a CCyR at 2 years was $<20 \%$ [8]. In addition to the non-achievement of time-based landmark responses, loss of a previously achieved response or progression to advanced phase disease should also trigger a change in treatment [35]. It should also be noted that the NCCN provides criteria that define partial resistance and suggests that treatment should be reassessed in these cases, even in absence of outright failure (suboptimum responses). In such cases, the patient may continue to benefit from the current treatment schedule, but longterm outcome may improve under an alternative strategy.

Evidence is now emerging to suggest that time-based response landmarks earlier than those proposed by the NCCN are warning signs in patients unlikely to achieve long-term benefits from imatinib, in particular the failure to achieve an early cytogenetic or molecular response. For example, patients in the IRIS trial who did not achieve a CCyR with imatinib by 12 months had a significantly higher risk of disease progression than patients with such a response [7]. Equally, not achieving a cytogenetic response at 3 or 6 months was associated with lower overall survival and PFS compared with patients who reached these responses [37, 39-42]. A recent retrospective study has showed that not achieving a MCyR by 6 months is predictive of decreased overall survival [13]. Nevertheless, some patients without achieving a CCyR may have disease control for years. Long-term, prospective studies are needed to define the optimum cues for TKI changes in the absence of overt resistance or progression.

In terms of molecular response, patients who fail to achieve a $1-\log$ reduction at 3 months, or a $>2-\log$ reduction by 6 months in BCR-ABL transcript levels are unlikely to subsequently achieve a substantial response and are at high risk for disease progression [24, 43]. Achievement of a MMR by 12 months appears to provide maximal protection from disease progression. Five-year follow-up data from the IRIS study revealed that no patient who achieved a MMR by 12 months had progressed to advanced disease [7]. Patients who had both a CCyR and MMR at 12 months of imatinib therapy had a $100 \%$ probability of remaining progression-free at 24 months as compared with $95 \%$ for patients who had a CCyR and a $<3-\log$ reduction of $\mathrm{BCR}-\mathrm{ABL}$ transcripts, and $85 \%$ for patients who did not achieve a CCyR [44]. However, the degree of molecular response in patients already in CCyR is not associated with differences in survival outcome [13]. In contrast, a rise in BCR-ABL transcripts can serve as an early indicator of the development of resistance, but a single test showing an increase in transcripts should not prompt a change in treatment [21, 45-47]. Emergence of imatinib-resistant BCR-ABL mutations at any time during treatment is equivalent to a diagnosis of disease progression and should prompt a change in therapy [35].

\section{Approved second-line TKI-based treatments: results from clinical trials in patients with imatinib-resistant or -intolerant CML}

Patients who fail first-line imatinib therapy should be considered for a change in treatment. Current guidelines for CML provide three options: high-dose imatinib, dasatinib, or nilotinib [35]. Key factors that may influence the choice of agent used are given in Table 4.

\section{High-dose imatinib}

Some mechanisms of imatinib resistance may be overcome by dose escalating imatinib. Certain BCR-ABL mutations confer intermediate levels of resistance to imatinib [50, 51]. Imatinib resistance caused by BCR-ABL overexpression may also be overcome using higher doses of imatinib [52]. Studies have shown that dose escalating imatinib can 
Table 4 Factors affecting the choice of second-line TKI for the treatment of CML [48, 49]

\begin{tabular}{|c|c|c|c|c|}
\hline Agent & Contraindications & Boxed warnings & Key warnings and precautions & $\begin{array}{l}\text { Key BCR-ABL mutations } \\
\text { (resistance or low efficacy) }\end{array}$ \\
\hline Imatinib & None & None & $\begin{array}{l}\text { - Edema and fluid retention } \\
\text { - Cytopenias } \\
\text { - CHF, left ventricular dysfunction } \\
\text { and cardiogenic shock } \\
\text { - Hepatotoxicity } \\
\text { - Hemorrhage } \\
\text { - Bullous dermatologic reactions }\end{array}$ & $\begin{array}{l}\text { >40; including P-loop (e.g., } \\
\text { Y253F/H, E255 K/V), } \\
\text { contact site (e.g., T315I), } \\
\text { SH2 binding site, and A-loop }\end{array}$ \\
\hline Dasatinib & None & None & $\begin{array}{l}\text { - Myelosuppression } \\
\text { - Bleeding related events } \\
\text { - Fluid retention } \\
\text { - QT prolongation } \\
\text { - Interactions with H2 blockers and PPIs }\end{array}$ & T315I, F317L \\
\hline Nilotinib & $\begin{array}{l}\text { Patients with } \\
\text { hypokalemia, } \\
\text { hypomagnesemia, } \\
\text { or long QT syndrome }\end{array}$ & $\begin{array}{l}\text { QT prolongation and } \\
\text { sudden deaths }\end{array}$ & $\begin{array}{l}\text { - Myelosuppression } \\
\text { - QT prolongation and sudden deaths } \\
\text { - Elevated serum lipase } \\
\text { - Hepatic function } \\
\text { - Electrolyte abnormalities } \\
\text { - Drug interactions } \\
\text { - Food effects }\end{array}$ & $\begin{array}{l}\text { T315I, P-loop mutations, } \\
\text { F359 }\end{array}$ \\
\hline
\end{tabular}

CHF congestive heart failure

induce responses in some patients who relapsed or were refractory to standard doses, with most benefit observed in patients who had suboptimal responses, while those who fail treatment rarely benefit [53, 54]. High-dose imatinib rarely results in deep, durable responses. Studies indicate that MCyRs are only gained by $26-38 \%$ of patients [53, 54]. Moreover, any responses gained are typically shortlived, best attained cytogenetic responses being soon lost by $43-50 \%$ of patients $[54,55]$. Furthermore, almost all (93\%) patients who do not achieve any cytogenetic response on standard-dose imatinib do not benefit from a high-dose regimen $[53,55]$. Also, many patients were intolerant of high-dose imatinib and dose reductions were required in $41 \%$ of patients [53].

\section{Dasatinib}

Dasatinib overcomes most forms of imatinib resistance. It has activity against all BCR-ABL point mutations except T315I and unlike imatinib, dasatinib can bind multiple conformations of BCR-ABL and is a potent inhibitor of the SFKs associated with BCR-ABL-independent imatinib resistance [56, 57]. In contrast to imatinib, dasatinib is not a substrate for P-glycoprotein (Pgp) efflux pump, and OCT-1 does not significantly influence dasatinib uptake [58]. Dasatinib has 325-fold greater potency versus imatinib against BCR-ABL and therefore may also overcome imatinib resistance mediated by increased expression of BCR-ABL [33, 34].

Dasatinib has been assessed in a phase II development program: Src-ABL Tyrosine Kinase Inhibition Activity Research Trials (START). Four single-arm trials were initiated in adult patients with imatinib-resistant or imatinib-intolerant leukemias: START-C (CP CML), START-A (AP CML), START-B (myeloid BC CML), and START-L (lymphoid $\mathrm{BC} \mathrm{CML}$ and $\mathrm{Ph}^{+} \mathrm{ALL}$ ). One prospective randomized trial, START-R, evaluated a dasatinib arm and a high-dose imatinib arm in patients who were previously resistant to standard-dose imatinib.

In patients with CP CML after 24 months of follow-up, dasatinib induced CHRs and MCyRs in 91 and 62\% of patients, respectively [59]. Most MCyRs observed were complete cytogenetic remissions, observed in over half of patients $(53 \%)$. MMRs were also achieved in $47 \%$ of patients [59]. With relatively limited follow-up responses seem to be durable. At 15 months, the PFS rate was 90 , and $97 \%$ of patients who had achieved a MCyR maintained it up to this time point [60]. After a 24-month follow-up, PFS was $80 \%$ (75\% in imatinib-resistant and $94 \%$ in imatinib-intolerant patients); $88 \%$ of patients who had achieved a MCyR having maintained it to this time point. Also at 24 months, OS was 94\% (92\% in imatinib-resistant and 100\% in imatinibintolerant patients) [59]. Dasatinib has also shown marked activity in patients with AP or BP CML [61, 62]. In patients with AP CML, the MCyR rate at 24 months was 40, and 61\% 
of patients achieving a MCyR maintained this response at 24 months. The median PFS was 19.5 months [63]. Though associated with a significantly lower response rate, deep and durable responses were also noted at 24 months in patients with BC CML [64]. But the majority of patients with BC CML do not have a sustained clinical benefit from dasatinib or other TKI-based therapy and should be considered for allogeneic stem cell transplantation.

The START-R study compared dasatinib with high-dose imatinib (800 mg/day) in patients with CP CML and resistance to imatinib 400-600 mg/day [65]. After 24 months of follow-up, dasatinib was superior to high-dose imatinib for rates of MCyR (53 vs. 33\%; $P=0.017)$, CCyR (44 vs. $18 \% ; P=0.003$ ), $\mathrm{MMR}(29$ vs. $12 \% ; P=0.028)$, and PFS (86 vs. $65 \% ; P=0.001$ ) [66]. Responses were also more durable in the dasatinib arm; $90 \%$ of patients receiving dasatinib maintained MCyR at 18 months compared with $74 \%$ of patients receiving imatinib [66].

In the START program, dasatinib demonstrated efficacy in patients with all imatinib-resistant BCR-ABL mutations tested, including P-loop mutations (where similar efficacy to wild type BCR-ABL is observed), except for T315 1 [61, $62,67]$. In the START-R study, higher rates of MCyR were observed in both mutation-positive and mutation-negative patients receiving dasatinib as compared with those receiving high-dose imatinib. Furthermore, only dasatinib induced responses in patients with P-loop mutations [65]. A report evaluating response to dasatinib by baseline $\mathrm{BCR}$ ABL mutation phenotype among patients enrolled in the START-C study confirmed activity across a range of mutants, including those in the P-loop, but also suggested that patients with F317L mutations may have diminished responses to dasatinib [68].

Dasatinib is generally well tolerated. Most adverse events (AEs) were grade 1-2 and resolved either spontaneously or with appropriate supportive care. Pleural effusions (all grades, 23\%; grade 3-4, 5\% at $70 \mathrm{mg}$ BID; all grades, 10\%; grade 3-4, 2\% at $100 \mathrm{mg}$ daily) and grade 3-4 cytopenias (neutropenia $46 \%$, thrombocytopenia $41 \%$, and anemia $18 \%$ ) can occur with dasatinib. These AEs can usually be managed with dose interruption or reduction [48]. Additionally, diuretics and/or steroids (prednisone $20 \mathrm{mg}$ po daily $\times 3$ days) and occasionally thoracentesis may be indicated in the management of dasatinib induced pleural effusions [35, 69]. Additionally, $10 \%$ of CP patients experienced hypophosphatemia in clinical studies [48]. There was no evidence of cumulative toxicity on long-term therapy [67]. As for all approved TKI treatments for CML, dasatinib is metabolized via the hepatic CYP3A4 cytochrome system. Care should therefore be taken when dasatinib is taken in conjunction with other agents that interact with this system.
Based on the data from the START program, dasatinib was approved for treated imatinib-resistant and -intolerant patients across all phases of CML (and $\mathrm{Ph}^{+} \mathrm{ALL}$ ) at a dosage of $70 \mathrm{mg}$ twice daily. Results from a recent phase III dose-optimization study recently prompted a change in the recommended daily dose for patients with $\mathrm{CP} C \mathrm{CML}$ [70].

The rationale for performing the dose-optimization study derived from two clinical observations. First, in the phase I study of dasatinib, CHRs and MCyRs were achieved at total daily doses of 100 and $140 \mathrm{mg}$ with both once daily and twice daily treatment regimens [71]. Second, the median delivered dose in the phase II program in CP CML patients was approximately $100 \mathrm{mg} /$ day. It was therefore decided to compare once and twice daily regimens at total daily doses of both 100 and $140 \mathrm{mg}$. After a minimum follow-up of 6 months, similar response rates were seen across all four dasatinib arms (CHRs in 8692\%; MCyRs in 54-59\%; CCyRs in 41-45\%). PFS at 6 months was $92 \%$ for dasatinib $100 \mathrm{mg}$ once daily and $89 \%$ for dasatinib $70 \mathrm{mg}$ twice daily [70]. No significant difference was apparent between regimens also after a follow-up of 12 months (MCyRs in 56-63\%) [70]. Twelve-month data also indicate that the $100 \mathrm{mg}$ once daily schedule also has activity across all BCR-ABL mutations, except T315I.

Differences between regimens evaluated in the doseoptimization study were apparent, however, in terms of safety [70]. In the $100 \mathrm{mg}$ once daily arm, there was a significantly lower frequency of grade 3-4 thrombocytopenia compared with the $70 \mathrm{mg}$ twice daily arm (22 vs. $37 \% ; P=0.004)$. Frequencies of anemia, leukocytopenia, and neutropenia were also less, but these reductions were not statistically significant $(P>0.05)$. Pleural effusions (all grades) also occurred less frequently with dasatinib $100 \mathrm{mg}$ once daily ( 7 vs. $16 \% ; P=0.028$ ). Improved safety with the $100 \mathrm{mg}$ once daily schedule was also reflected by the lower incidences of dose interruption (51 vs. $68 \%$ ), reduction (30 vs. $55 \%$ ), and discontinuation (16 vs. $23 \%$ ) relative to the $70 \mathrm{mg}$ twice daily schedule.

Results of this study demonstrate that, compared with the previously recommended $70 \mathrm{mg}$ twice daily dose, a $100 \mathrm{mg}$ once daily regimen offers a more favorable overall benefit-risk assessment in chronic phase CML. A separate trial in advanced phase patients showed that a once daily dose had a better safety profile with similar response rates; however, further follow-up is necessary before a change in treatment practice can be recommended [72]. The current prescribing information for dasatinib now recommends regimens of $100 \mathrm{mg}$ once daily for the treatment of $\mathrm{CP}$ CML and $70 \mathrm{mg}$ twice daily for the treatment of AP or BC CML, and $\mathrm{Ph}^{+} \mathrm{ALL}$ [48]. 


\section{Nilotinib}

Nilotinib is an analog of imatinib, 10- to 50-fold more potent than its parent compound against BCR-ABL, which has recently been approved for the treatment of patients with $\mathrm{CP}$ or AP CML who have failed prior treatment. In vitro evidence shows that nilotinib has activity against all imatinib-resistant BCR-ABL mutations except T315 1, but that activity against P-loop mutations and other imatinibresistant mutations, including F359C, may be diminished $[56,73]$. Inhibitory plasma concentrations of nilotinib can usually be obtained for the majority of P-loop mutations and further in vivo evidence is needed.

Second-line nilotinib $800 \mathrm{mg} /$ day has been assessed in an open-label phase II trial in patients with CP CML [74]. After 18 months of follow-up, CHRs were achieved in $85 \%$ of patients, MCyRs in $57 \%$, and CCyRs in $41 \%$. In total, $84 \%$ of patients who achieved a MCyR maintained it for 18 months [75]. A time to progression analysis showed that $64 \%$ of patients had not progressed at 18 months [75]. The overall survival rate at this time point was $91 \%$ [75]. These results are similar to those seen with dasatinib in the START-C trial.

Activity of nilotinib $800 \mathrm{mg} /$ day in patients with AP CML was demonstrated in a phase II study [76]. After a follow-up of at least 12 months, MCyRs were achieved in $31 \%$ of patients [77]. At 12 months the percentage of patients who were progression-free was $57 \%$, and the overall survival rate was $81 \%$.

In the pivotal phase II study in patients with CP CML, nilotinib showed activity in patients harboring many imatinib-resistant BCR-ABL mutations (except T315I), and also in patients with resistance not associated with BCR-ABL mutations [74]. However, a sub-analysis of this study of the occurrence of baseline BCR-ABL mutations and their effect on treatment outcome after 12 months of treatment showed that no patient with $\mathrm{Y} 253 \mathrm{H}, \mathrm{E} 255 \mathrm{~K} / \mathrm{V}$, or $\mathrm{F} 359 \mathrm{C} / \mathrm{V}$ mutations achieved a CCyR; though the number of patients with each mutation is relatively low, making definitive conclusions difficult [64]. These findings were supported in evaluations of patients enrolled in phase II clinical studies of nilotinib, which also showed that $\mathrm{Y} 253 \mathrm{~F} / \mathrm{H}, \mathrm{E} 255 \mathrm{~K}$, and E255K/V mutations were associated with disease progression [78, 79]. The P-loop mutations $\mathrm{Y} 253 \mathrm{H}$ and E255 K/V are also among those that most frequently develop during nilotinib treatment and are associated with disease progression [78, 80].

The AEs associated with nilotinib therapy are predominantly mild to moderate in severity [74]. The incidences of grade 3-4 thrombocytopenia and neutropenia were similar to that observed with the dasatinib $100 \mathrm{mg}$ once daily [77]. Nilotinib's specificity for the ABL kinase and comparably minimal inhibition of c-KIT and PDGFR also alters its toxicity profile. Inhibition of PDGFR is thought to be the cause of the edema associated with imatinib and dasatinib therapy [81]. In clinical trials only $10 \%$ of patients taking nilotinib have had peripheral edema and none have had grade 3-4 events [82]. There is minimal cross-intolerance with imatinib, although approximately half (49\%) the patients with hematologic intolerance to imatinib experienced a recurrence of the grade 3-4 hematologic event (mostly thrombocytopenia) during nilotinib therapy [83].

Notably, the prescribing information for nilotinib contains a black box warning regarding the risk of QTc prolongation and sudden death. It should be noted that prolongation of QTc occurs with both imatinib and dasatinib, although sudden death was not observed with these agents in clinical trials.

QTc prolongation was observed on clinical studies with nilotinib and sudden deaths occurred that were believed to be related to ventricular repolarization abnormalities. If QTc prolongation does occur, treatment should be interrupted; dose reductions or discontinuation may also be necessary. Additionally, as with all ABL TKIs concomitant medication should be reviewed [82].

Nilotinib treatment may also be associated with biochemical abnormalities. Increases in serum lipase, bilirubin, alanine or aspartate aminotransferases, and alkaline phosphatase have been reported [82]. If these AEs occur, treatment should be withheld until serum levels return to grade $\leq 1$. Treatment can then be resumed at a reduced dosage $(400 \mathrm{mg} /$ day). Hyperglycemia and electrolyte abnormalities [i.e., hypophosphatemia (10\%), hypokalemia $(1 \%)$, hyperkalemia $(4 \%)$, hypocalcemia (1\%), and hyponatremia (3\%)] can also occur with nilotinib [82]. However, these electrolyte abnormalities may also occur with dasatinib in a similar proportion of patients [48, 82]. The prescribing information for nilotinib also carries contraindications for hypokalemia and hypomagnesemia due to these abnormalities potential worsening of QTc prolongation [82]. Nilotinib, like imatinib and dasatinib, is metabolized via the hepatic CYP3A4 cytochrome system, and caution should be taken when dasatinib is administered in conjunction with other compounds which interact with this system.

\section{When to switch from imatinib to second-line TKI}

Data from clinical trials has demonstrated that second-line TKIs are most effective in extending survival when administered in CP rather than in the advanced phases of CML. Three-year survival rates for imatinib-resistant patients decrease as a function of disease status; $72 \%$ for $\mathrm{CP}, 30 \%$ for AP, and $7 \%$ for BC [12]. Furthermore, PFS was improved when dasatinib was used upon loss of 
cytogenetic response during imatinib treatment versus loss of hematologic response [84]. Current consensus guidelines recommend switching from imatinib to a secondgeneration TKI if (1) a CHR is not achieved by 3 months, (2) no cytogenetic response by 6 months, (3) minor or no cytogenetic response is achieved by 12 months, (4) a partial cytogenetic response is not achieved by 18 months, or (5) on disease progression to AP or BC CML.

\section{Use of second-line TKIs in the first-line}

Given the clinical benefit gained with dasatinib and nilotinib following imatinib failure, the possibility arises that earlier and deeper responses could be achieved with firstline use. Current evidence demonstrates that responses in patients with CP CML are greater than those in patients with advanced disease [12]. Moreover, patients in late CP are at greater risk of progression than patients with early CP CML. The contribution of BCR-ABL mutations during therapy is also an important issue as genetic instability increases with progression, making the disease more difficult to treat. As second-line agents are effective against a larger spectrum of BCR-ABL mutations than imatinib, the emergence of resistance may decrease if these agents are used earlier. Two clinical trials based at the M.D. Anderson Cancer Centre in Houston, Texas are currently evaluating first-line therapy with these agents. Early data for a small number of patients are available.

First-line dasatinib (100 mg/day) administered on a once- or twice-daily regimen is being investigated $(n=40$; accrual ongoing) [85]. Preliminary results show that CCyRs were achieved in $94 \%$ of patients at 6 months, and in all evaluable patients $(100 \%)$ at 12 months. Nilotinib $800 \mathrm{mg} /$ day elicited similar activity [86]; CCyR rates at 6 and 12 months were $100 \%$. Both drugs elicited significantly $(P<0.001)$ deeper responses at these time points than imatinib (historical controls) [85, 86]. Marked superiority over imatinib in this setting can also be demonstrated by comparing the response rates discussed above with those obtained for first-line imatinib in the IRIS study; after 18 months of follow-up in this trial the CCyR rate was 74\% [9]. But until these trials are reported with sufficient followup, imatinib remains the standard of care for the first-line management of CP CML.

\section{Third-line tyrosine kinase inhibitors}

Resistance and intolerance to currently approved agents have necessitated the development of further compounds for the treatment of CML. Of note, there is a particular need for agents that are active in patients carrying the T315I BCR-ABL mutation, which is resistant to imatinib and both second-line TKIs [56]. Selected major developments are discussed below; a comprehensive review is beyond the scope of this article.

Bosutinib (SKI-606) inhibits both BCR-ABL and Src, being 10- to 20 -fold more potent against BCR-ABL than imatinib in vitro, but has no activity against the T315I mutation [87, 88]. From preliminary data from a study in imatinib-resistant patients with CP CML, CCyRs were achieved by $30 \%$ of patients [89]. Activity in advanced stage CML and $\mathrm{Ph}^{+} \mathrm{ALL}$ has also been demonstrated [90]. A phase III comparison of bosutinib and imatinib in newly diagnosed patients with CML is currently in recruitment.

INNO-406 is another dual BCR-ABL and SFK inhibitor, 55 -fold more potent than imatinib in vitro, but also has no activity against T315I [91]. However, activity including CCyRs has been observed in an ongoing phase I dosefinding study in patients with CML (any phase) or $\mathrm{Ph}^{+} \mathrm{ALL}$ resistant or intolerant to first- or second-line treatment, including dasatinib or nilotinib [92]. Aurora kinase inhibitors are a new class of compound that may provide successful treatment for patients with T315I-mutated CML. PHA-739538 is an aurora kinase inhibitor with strong antiproliferative activity against CD34+ cells taken from untreated CML patients and also from imatinib-resistant patients, including those with T315I [93]. A phase II study in patients with CML is now in progress [94]. Seven patients are currently enrolled, six of whom have T315I mutations. To date, two patients with T315I mutations have achieved cytogenetic responses, one of them a CCyR. The development of another aurora kinase inhibitor, MK-0457, was recently stopped following concerns over cardiotoxicity. Omacetaxine mepesuccinate (homoharringtonine; HHT) is a multi-targeted protein synthase inhibitor. This compound is currently in phase II development in imatinibresistant patients with CML (all phases) and who carry T315I-mutated BCR-ABL [95]. To date, 29 patients have been enrolled, 17 of which have CP CML. Preliminary data indicate a CHR rate of $45 \%$ in CP CML patients. Cytogenetic responses have been reported in $27 \%$ of patients with CP CML; two responses were CCyRs. Reversion of T315I status in some patients has also been observed.

Finally, the addition of rapamycin to current TKI therapy may constitute a novel approach to the treatment of patients with refractory disease. The mammalian target of rapamycin (mTOR) is constitutively activated in BCRABL-transformed cells, and rapamycin inhibits the growth of CML cells obtained from patients with imatinib-resistant disease [96]. Recently, hematologic activity for this compound has been demonstrated in leukemic patients [97]. 


\section{Conclusions}

Targeted therapy with TKIs has dramatically changed the prognosis for patients with CML. Despite the impressive activity of imatinib, resistance to this therapy has become a significant clinical issue. Dasatinib and nilotinib are both effective in patients following imatinib failure or intolerance. High-dose imatinib may be effective in some patients following resistance to standard-dose imatinib, but dasatinib has been shown to be more efficacious. Careful consideration should be taken when deciding which TKI to select following imatinib failure. Currently, there is no definitive evidence for the superiority of either dasatinib or nilotinib in CP or AP CML. Dasatinib is the preferred agent in BC CML. Treatment decisions should be directed by both efficacy and safety parameters (Table 4). In particular, based on pre-clinical and clinical data, patients with certain P-loop mutations such as Y253F, E255V, and F359 may respond better to dasatinib; in contrast, patients with the point mutation F317L may respond better to nilotinib. Patients at risk for complications from fluid retention may be better suited to nilotinib. For each of these second-line TKIs, it may be more beneficial to administer them earlier in the disease course in order to minimize the emergence of resistance and improve the overall duration of response. Trials are currently underway to evaluate this hypothesis.

Acknowledgments Writing and editorial support were provided by Johnathan Maher and Josh Collis, funded by Bristol-Myers Squibb. Bristol-Myers Squibb did not provide honoraria to the authors.

Conflict of interest statement Writing and editorial support were provided by Johnathan Maher and Josh Collis, funded by BristolMyers Squibb.

Open Access This article is distributed under the terms of the Creative Commons Attribution Noncommercial License which permits any noncommercial use, distribution, and reproduction in any medium, provided the original author(s) and source are credited.

\section{References}

1. Faderl S, Talpaz M, Estrov Z, O'Brien S, Kurzrock R, Kantarjian HM (1999) The biology of chronic myeloid leukemia. N Engl J Med 341:164-172

2. Sawyers CL (1999) Chronic myeloid leukemia. N Engl J Med 340:1330-1340

3. DeVita VT, Hellman S, Rosenburg SA (2001) Cancer. Principles and practice of oncology, 6th edn. Lippincott Williams and Wilkins, Philadelphia

4. Savage DG, Szydlo RM, Chase A et al (1997) Bone marrow transplantation for chronic myeloid leukaemia: the effects of differing criteria for defining chronic phase on probabilities of survival and relapse. Br J Haematol 99:30-35

5. Jaffe ES, Harris NL, Stein H, Vardiman JW (2001) WHO classification of tumours. Pathology and genetics of tumours of haematopoietic and lymphoid tissues. IARC Press, France
6. CML Trialists' Collaborative Group (1997) Interferon alfa versus chemotherapy for chronic myeloid leukemia: a meta-analysis of seven randomized trials: Chronic Myeloid Leukemia Trialists' Collaborative Group. J Natl Cancer Inst 89:1616-1620

7. Druker BJ, Guilhot F, O'Brien SG et al (2006) Five-year followup of patients receiving imatinib for chronic myeloid leukemia. N Engl J Med 355:2408-2417

8. Baccarani M, Saglio G, Goldman J et al (2006) Evolving concepts in the management of chronic myeloid leukemia: recommendations from an expert panel on behalf of the European Leukemianet. Blood 108:1809-1820

9. O'Brien SG, Guilhot F, Larson RA et al (2003) Imatinib compared with interferon and low-dose cytarabine for newly diagnosed chronic-phase chronic myeloid leukemia. N Engl J Med 348:994-1004

10. Sawyers CL, Hochhaus A, Feldman E et al (2002) Imatinib induces hematologic and cytogenetic responses in patients with chronic myelogenous leukemia in myeloid blast crisis: results of a phase II study. Blood 99:3530-3539

11. Talpaz M, Silver RT, Druker BJ et al (2002) Imatinib induces durable hematologic and cytogenetic responses in patients with accelerated phase chronic myeloid leukemia: results of a phase 2 study. Blood 99:1928-1937

12. Kantarjian H, O'Brien S, Talpaz M et al (2007) Outcome of patients with Philadelphia chromosome-positive chronic myelogenous leukemia post-imatinib mesylate failure. Cancer 109:1556-1560

13. Kantarjian H, O'Brien S, Shan J et al (2008) Cytogenetic and molecular responses and outcome in chronic myelogenous leukemia: need for new response definitions? Cancer 112:837-845

14. Hughes T, Branford S (2006) Molecular monitoring of BCR-ABL as a guide to clinical management in chronic myeloid leukaemia. Blood Rev 20:29-41

15. de Lavallade H, Apperley JF, Khorashad JS et al (2008) Imatinib for newly diagnosed patients with chronic myeloid leukemia: incidence of sustained responses in an intention-to-treat analysis. J Clin Oncol 26:3358-3363

16. Steegman JL, Michallet M, Morra E et al (2008) Imatinib use in chronic phase CML in clinical practice: the UNIC study. J Clin Oncol 26:7077a

17. Hochhaus A, Erben P, Ernst T, Mueller MC (2007) Resistance to targeted therapy in chronic myelogenous leukemia. Semin Hematol 44(Suppl 1):15-24

18. Hochhaus A, La Rosee P (2004) Imatinib therapy in chronic myelogenous leukemia: strategies to avoid and overcome resistance. Leukemia 18:1321-1331

19. Shah NP, Nicoll JM, Nagar B et al (2002) Multiple BCR-ABL kinase domain mutations confer polyclonal resistance to the tyrosine kinase inhibitor imatinib (STI571) in chronic phase and blast crisis chronic myeloid leukemia. Cancer Cell 2:117-125

20. Soverini S, Colarossi S, Gnani A et al (2006) Contribution of ABL kinase domain mutations to imatinib resistance in different subsets of Philadelphia-positive patients: by the GIMEMA Working Party on Chronic Myeloid Leukemia. Clin Cancer Res 12:7374-7379

21. Hughes TP, Deininger MW, Hochhaus A et al (2006) Monitoring CML patients responding to treatment with tyrosine kinase inhibitors: review and recommendations for 'harmonizing' current methodology for detecting BCR-ABL transcripts and kinase domain mutations and for expressing results. Blood 108:28-37

22. Deininger M, Buchdunger E, Druker BJ (2005) The development of imatinib as a therapeutic agent for chronic myeloid leukemia. Blood 105:2640-2653

23. Branford S, Rudzki Z, Walsh S et al (2003) Detection of BCR$\mathrm{ABL}$ mutations in patients with CML treated with imatinib is virtually always accompanied by clinical resistance, and 
mutations in the ATP phosphate-binding loop (P-loop) are associated with a poor prognosis. Blood 102:276-283

24. La Rosee P, Corbin AS, Stoffregen EP et al (2002) Activity of the Bcr-Abl kinase inhibitor PD180970 against clinically relevant $\mathrm{Bcr}-\mathrm{Abl}$ isoforms that cause resistance to imatinib mesylate (Gleevec, STI571). Cancer Res 62:7149-7153

25. Jabbour E, Kantarjian H, Jones D et al (2008) Characteristics and outcomes of patients with chronic myeloid leukemia and T315I mutation following failure of imatinib mesylate therapy. Blood 112:53-55

26. Meyn III MA, Wilson MB, Abdi FA et al (2006) Src family kinases phosphorylate the $\mathrm{Bcr}-\mathrm{Abl} \mathrm{SH} 3-\mathrm{SH} 2$ region and modulate Bcr-Abl transforming activity. J Biol Chem 281:30907-30916

27. Lionberger JM, Wilson MB, Smithgall TE (2000) Transformation of myeloid leukemia cells to cytokine independence by Bcr-Abl is suppressed by kinase-defective Hck. J Biol Chem 275:1858118585

28. Donato NJ, Wu JY, Stapley J et al (2003) BCR-ABL independence and LYN kinase overexpression in chronic myelogenous leukemia cells selected for resistance to STI571. Blood 101:690 698

29. Donato NJ, Wu JY, Stapley J et al (2004) Imatinib mesylate resistance through $\mathrm{BCR}-\mathrm{ABL}$ independence in chronic myelogenous leukemia. Cancer Res 64:672-677

30. Ban K, Gao Y, Amin HM et al (2008) BCR-ABL1 mediates upregulation of Fyn in chronic myelogenous leukemia. Blood 111:2904-2908

31. Zong Y, Zhou S, Sorrentino BP (2005) Loss of P-glycoprotein expression in hematopoietic stem cells does not improve responses to imatinib in a murine model of chronic myelogenous leukemia. Leukemia 19:1590-1596

32. Thomas J, Wang L, Clark RE, Pirmohamed M (2004) Active transport of imatinib into and out of cells: implications for drug resistance. Blood 104:3739-3745

33. Gorre ME, Mohammed M, Ellwood K et al (2001) Clinical resistance to STI-571 cancer therapy caused by BCR-ABL gene mutation or amplification. Science 293:876-880

34. Hochhaus A, Kreil S, Corbin AS et al (2002) Molecular and chromosomal mechanisms of resistance to imatinib (STI571) therapy. Leukemia 16:2190-2196

35. National Comprehensive Cancer Network (NCCN): clinical practice guidelines in oncologyTM: chronic myelogenous leukemia, V.1.2008. Available via http://www.nccn.org/professionals/ physician_gls/PDF/cml.pdf

36. Roy L, Guilhot J, Krahnke T et al (2006) Survival advantage from imatinib compared to the combination interferon-alpha plus cytarabine in chronic phase CML: historical comparison between two phase III trials. Blood 108:1478-1484

37. Kantarjian HM, O'Brien S, Cortes JE et al (2003) Complete cytogenetic and molecular responses to interferon-alpha-based therapy for chronic myelogenous leukemia are associated with excellent long-term prognosis. Cancer 97:1033-1041

38. Hughes T (2006) ABL kinase inhibitor therapy for CML: baseline assessments and response monitoring. Hematol Am Soc Hematol Educ Program 211-218

39. Kantarjian H, Talpaz M, O'Brien S et al (2004) High-dose imatinib mesylate therapy in newly diagnosed Philadelphia chromosome-positive chronic phase chronic myeloid leukemia. Blood 103:2873-2878

40. Marin D, Marktel S, Bua M et al (2003) Prognostic factors for patients with chronic myeloid leukaemia in chronic phase treated with imatinib mesylate after failure of interferon alfa. Leukemia 17:1448-1453

41. O'Brien S, Tefferi A, Valent P (2004) Chronic myelogenous leukemia and myeloproliferative disease. Hematol Am Soc Hematol Educ Program 146-162
42. Gambacorti C, Talpaz M, Sawyers CL et al (2005) Five year follow-up results of a phase II trial in patients with late chronic phase (L-CP) chronic myeloid leukemia (CML) treated with imatinib who are refractory/intolerant of interferon-alpha. Blood 106:1089a

43. Quintas-Cardama A, Kantarjian HM, Jones D et al (2006) Delayed achievement of molecular responses is associated with increased risk of progression among patients (pts) with chronic myelogenous leukemia (CML) in chronic phase (CP) treated with imatinib (IM). Blood 108:432a

44. Hughes TP, Kaeda J, Branford S et al (2003) Frequency of major molecular responses to imatinib or interferon alfa plus cytarabine in newly diagnosed chronic myeloid leukemia. N Engl J Med 349:1423-1432

45. Goldman JM (2007) How I treat chronic myeloid leukemia in the imatinib era. Blood 110:2828-2837

46. Martinelli G, Iacobucci I, Rosti G et al (2006) Prediction of response to imatinib by prospective quantitation of $\mathrm{BCR}-\mathrm{ABL}$ transcript in late chronic phase chronic myeloid leukemia patients. Ann Oncol 17:495-502

47. Barnes DJ, Palaiologou D, Panousopoulou E et al (2005) Bcr-Abl expression levels determine the rate of development of resistance to imatinib mesylate in chronic myeloid leukemia. Cancer Res 65:8912-8919

48. Bristol-Myers Squibb Company (2007) Dasatinib package insert. Bristol-Myers Squibb Company. Princeton, NJ

49. Novartis Pharmaceuticals Corporation (2007) Gleevec ${ }^{\mathrm{TM}}$ (imatinib mesylate) package insert. East Hanover, NJ

50. Roumiantsev S, Shah NP, Gorre ME et al (2002) Clinical resistance to the kinase inhibitor STI-571 in chronic myeloid leukemia by mutation of Tyr-253 in the Abl kinase domain P-loop. Proc Natl Acad Sci USA 99:10700-10705

51. Azam M, Latek RR, Daley GQ (2003) Mechanisms of autoinhibition and STI-571/imatinib resistance revealed by mutagenesis of BCR-ABL. Cell 112:831-843

52. Kantarjian HM, Talpaz M, Giles F et al (2006) New insights into the pathophysiology of chronic myeloid leukemia and imatinib resistance. Ann Intern Med 145:913-923

53. Kantarjian HM, Talpaz M, O'Brien S et al (2003) Dose escalation of imatinib mesylate can overcome resistance to standard-dose therapy in patients with chronic myelogenous leukemia. Blood 101:473-475

54. Zonder JA, Pemberton P, Brandt $\mathrm{H}$ et al (2003) The effect of dose increase of imatinib mesylate in patients with chronic or accelerated phase chronic myelogenous leukemia with inadequate hematologic or cytogenetic response to initial treatment. Clin Cancer Res 9:2092-2097

55. Marin D, Goldman JM, Olavarria E, Apperley JF (2003) Transient benefit only from increasing the imatinib dose in CML patients who do not achieve complete cytogenetic remissions on conventional doses. Blood 102:2702-2703

56. O'Hare T, Walters DK, Stoffregen EP et al (2005) In vitro activity of Bcr-Abl inhibitors AMN107 and BMS-354825 against clinically relevant imatinib-resistant Abl kinase domain mutants. Cancer Res 65:4500-4505

57. Shah NP, Tran C, Lee FY et al (2004) Overriding imatinib resistance with a novel ABL kinase inhibitor. Science 305:399_ 401

58. Hiwase DK, White DL, Saunders VA et al (2007) In contrast to imatinib, OCT-1 mediated influx has minimal impact on cellular uptake of dasatinib in CML patients at diagnosis. Blood 110:575$576 a$

59. Mauro MJ, Baccarani M, Cervantes F et al (2008) Dasatinib 2-year efficacy in patients with chronic-phase chronic myelogenous leukemia (CML-CP) with resistance or intolerance to imatinib (START-C). J Clin Oncol 26(Suppl):7009a 
60. Hochhaus A, Mueller M, Cortes JE et al (2008) Dasatinib efficacy by dosing schedule across individual baseline BCR-ABL mutations in chronic phase chronic myelogenous leukemia (CML-CP) after imatinib failure. J Clin Oncol 26:7014a

61. Cortes J, Rousselot P, Kim DW et al (2007) Dasatinib induces complete hematologic and cytogenetic responses in patients with imatinib-resistant or -intolerant chronic myeloid leukemia in blast crisis. Blood 109:3207-3213

62. Guilhot F, Apperley J, Kim DW et al (2007) Dasatinib induces significant hematologic and cytogenetic responses in patients with imatinib-resistant or -intolerant chronic myeloid leukemia in accelerated phase. Blood 109:4143-4150

63. Rea D, Dombret H, Kim DW et al (2008) Dasatinib efficacy in patients with imatinib-resistant/-intolerant chronic myeloid leukemia in accelerated phase 24-month data from START-A. Haematologica 93(Suppl 1):391

64. Saglio G, Dombret H, Rea D et al (2008) Dasatinib efficacy in patients with imatinib-resistant/-intolerant chronic myeloid leukemia in blast phase: 24-month data from the START program. Haematologica 93(Suppl 1):S349

65. Kantarjian H, Pasquini R, Hamerschlak N et al (2007) Dasatinib or high-dose imatinib for chronic-phase chronic myeloid leukemia after failure of first-line imatinib: a randomized phase 2 trial. Blood 109:5143-5150

66. Rousselot PH, Facon T, Paquette R, Bleickardt D, Kantarjian HM (2008) Dasatinib or high-dose imatinib for patients with chronic myelogenous leukemia chronic-phase (CML-CP) resistant to standard-dose imatinib: 2-year follow-up data from START-R. J Clin Oncol 26(Suppl):S7012a

67. Hochhaus A, Kantarjian HM, Baccarani M et al (2007) Dasatinib induces notable hematologic and cytogenetic responses in chronic phase chronic myeloid leukemia after failure of imatinib therapy. Blood 109:2303-2309

68. Mueller MC, Erben P, Ernst T et al (2007) Molecular response according to type of preexisting BCR-ABL mutations after second line dasatinib therapy in chronic phase CML patients. Blood $110: 319 a$

69. Quintas-Cardama A, Kantarjian H, Munden R et al (2006) Pleural effusion in patients (pts) with chronic myelogenous leukemia (CML) treated with dasatinib after imatinib failure. Blood 108:2164a

70. Shah NP, Kantarjian HM, Kim DW et al (2008) Intermittent target inhibition with dasatinib $100 \mathrm{mg}$ once daily preserves efficacy and improves tolerability in imatinib-resistant and -intolerant chronic-phase chronic myeloid leukemia. J Clin Oncol 26:3204-3212

71. Talpaz M, Shah NP, Kantarjian H et al (2006) Dasatinib in imatinib-resistant Philadelphia chromosome-positive leukemias. N Engl J Med 354:2531-2541

72. Dombret H, Ottoman O, Goh Y et al (2007) Dasatinib $140 \mathrm{mg}$ QD vs $70 \mathrm{mg}$ BID in advanced-phase CML or Ph+ALL resistant or intolerant to imatinib: results from a randomized, phase-III trial (CA180035). Haematologica 92(Suppl 2):0859a

73. Weisberg E, Manley PW, Breitenstein W et al (2005) Characterization of AMN107, a selective inhibitor of native and mutant Bcr-Abl. Cancer Cell 7:129-141

74. Kantarjian HM, Giles F, Gattermann N et al (2007) Nilotinib (formerly AMN107), a highly selective Bcr-Abl tyrosine kinase inhibitor, is effective in patients with Philadelphia chromosomepositive chronic myelogenous leukemia in chronic phase following imatinib resistance and intolerance. Blood 110:3540-3546

75. Kantarjian HM, Giles FJ, Hochhaus A et al (2008) Nilotinib in patients with imatinib-resistant or -intolerant chronic myelogenous leukemia in chronic phase (CML-CP): updated phase II results. J Clin Oncol 26:7010a
76. le Coutre P, Ottmann OG, Giles F et al (2008) Nilotinib (formerly AMN107), a highly selective BCR-ABL tyrosine kinase inhibitor, is active in patients with imatinib-resistant or -intolerant accelerated-phase chronic myelogenous leukemia. Blood 111: 1834-1839

77. le Coutre P, Giles FJ, Apperley J et al (2008) Nilotinib in accelerated phase chronic myelogenous leukemia (CML-AP) patients with imatinib-resistance or -intolerance: update of a phase II study. J Clin Oncol 26:7050a

78. Branford S, Shou Y, Lawrence R, Rudzki Z, Hughes T (2007) The P-loop mutations $\mathrm{Y} 253 \mathrm{H}$ and E255K/V may develop more frequently than T3151 during nilotinib therapy after imatinib failure and are associated with progression in patients with Ph-positive leukemia. Haematologica 92(Suppl 2):S0904a

79. Hughes T, Saglio G, Martinelli G et al (2007) Responses and disease progression in CML-CP patients treated with nilotinib after imatinib failure appear to be affected by the BCR-ABL mutation status and types. Blood 110:320a

80. Cortes J, Jabbour E, Kantarjian H et al (2007) Dynamics of BCRABL kinase domain mutations in chronic myeloid leukemia after sequential treatment with multiple tyrosine kinase inhibitors. Blood 110:4005-4011

81. Deininger M (2008) Nilotinib. Clin Cancer Res 14:4027-4031

82. Novartis Pharmaceuticals Corporation (2007) Tasigna (nilotinib) prescribing information. East Hanover, NJ

83. Cortes J, Jabbour E, Hochhaus A et al (2007) Nilotinib is associated with minimal cross intolerance to imatinib in patients with imatinib-intolerant Philadelphia-positive $(\mathrm{Ph}+)$ chronic myelogenous leukemia (CML) in either chronic phase (CP) or accelerated phase (AP). Blood 110:1040a

84. Kantajarian HM, Quintas-Cardama A, O'Brien S et al (2007) Importance of early intervention with dasatinib at cytogenetic rather than hematologic resistance to imatinib. Blood 110:1036a

85. Borthakur G, Kantarjian HM, O'Brien SM et al (2008) Efficacy of dasatinib in patients (pts) with previously untreated chronic myelogenous leukemia (CML) in early chronic phase (CML-CP). $\mathrm{J}$ Clin Oncol 26:7013a

86. Cortes JE, O’Brien SM, Ferrajoli A et al (2008) Efficacy of nilotinib (AMN107) in patients (Pts) with newly diagnosed, previously untreated Philadelphia chromosome ( $\mathrm{Ph}$ )-positive chronic myelogenous leukemia in early chronic phase (CMLCP). J Clin Oncol 26(15S):7016a

87. Golas JM, Arndt K, Etienne C et al (2003) SKI-606, a 4-anilino3-quinolinecarbonitrile dual inhibitor of Src and Abl kinases, is a potent antiproliferative agent against chronic myelogenous leukemia cells in culture and causes regression of K562 xenografts in nude mice. Cancer Res 63:375-381

88. Puttini M, Coluccia AM, Boschelli F et al (2006) In vitro and in vivo activity of SKI-606, a novel Src-Abl inhibitor, against imatinib-resistant Bcr-Abl+ neoplastic cells. Cancer Res 66:11314-11322

89. Bruemmendorf TH, Cervantes F, Kim D et al (2008) Bosutinib is safe and active in patients (pts) with chronic phase (CP) chronic myeloid leukemia (CML) with resistance or intolerance to imatinib and other tyrosine kinase inhibitors. J Clin Oncol 26:7001a

90. Gambacorti-Passerini C, Kantarjian HM, Baccarani $M$ et al (2008) Activity and tolerance of bosutinib in patients with AP and BP CML and Ph+ALL. J Clin Oncol 26:7049a

91. Kimura S, Naito H, Segawa H et al (2005) NS-187, a potent and selective dual Bcr-Abl/Lyn tyrosine kinase inhibitor, is a novel agent for imatinib-resistant leukemia. Blood 106:3948-3954

92. Pinilla-Ibarz J, Kantarjian HM, Cortes JE et al (2008) A phase I study of INNO-406 in patients with advanced Philadelphia chromosome-positive $(\mathrm{Ph}+)$ leukemias who are resistant or 
intolerant to imatinib and may have also failed second-generation tyrosine kinase inhibitors. J Clin Oncol 26:7018a

93. Gontarewicz A, Balabanov S, Keller G et al (2008) Simultaneous targeting of Aurora kinases and Bcr-Abl kinase by the small molecule inhibitor PHA-739358 is effective against imatinibresistant BCR-ABL mutations including T315I. Blood 111:43554364

94. Paquette RL, Shah NP, Sawyers CL et al (2007) PHA-739358, an aurora kinase inhibitor, induces clinical responses in chronic myeloid leukemia harboring T315I mutations of BCR-ABL. Blood 110:1030a
95. Benichou A, Khoury HJ, Corm S et al (2008) Multicenter open label study of subcutaneous (SC) omacetaxine (OMA) in imatinib (IM)-resistant chronic myeloid leukemia (CML) patients (Pts) with the T315I mutation. J Clin Oncol 26:7021a

96. Mayerhofer M, Aichberger KJ, Florian S et al (2005) Identification of mTOR as a novel bifunctional target in chronic myeloid leukemia: dissection of growth-inhibitory and VEGF-suppressive effects of rapamycin in leukemic cells. FASEB J 19:960-962

97. Sillaber C, Mayerhofer M, Bohm A et al (2008) Evaluation of antileukaemic effects of rapamycin in patients with imatinibresistant chronic myeloid leukaemia. Eur J Clin Invest 38:43-52 